\title{
DISEÑO DE UNA PLANTA INDUSTRIAL PARA LA REUTILIZACIÓN DE LOS EFLUENTES GENERADOS EN LA PRODUCCIÓN DE CUERO
}

\section{DESIGN OF AN INDUSTRIAL PLANT FOR THE REUSE OF EFFLUENTS GENERATED IN LEATHER PRODUCTION}

\author{
G. Espinoza Gutiérrez ${ }^{1 *}$, P. Barreda Cuellar ${ }^{1 * *}$ y J. Aguilar Franco ${ }^{1}$ \\ ${ }^{1}$ Universidad Católica San Pablo. Instituto de Energía y Medio Ambiente. Arequipa, Perú. \\ *gisel.espinoza@ucsp.edu.pe \\ **paola.barreda@ucsp.edu.pe
}

(recibido/received: 21-Febrero-2019; aceptado/accepted: 11-Noviembre-2019)

\section{RESUMEN}

En el presente artículo se desarrolló el diseño e implementación de una planta industrial para el tratamiento de los efluentes generados en el proceso productivo de una curtiembre con el objetivo de lograr la reutilización de los mismos en sus actividades. Actualmente la empresa no se presentan mayores avances respecto al manejo de sus efluentes, siendo estos dispuestos directamente al sistema de alcantarillado lo que ha ocasionado el incumplimiento de la normativa ambiental vigente en el país provocando un impacto negativo en el medio ambiente. Debido a esto se decidió desarrollar un estudio técnico que permita implementar una planta de tratamiento adecuada las instalaciones y necesidades de la empresa. El sistema de tratamiento está dividido en tres unidades, la primera correspondiente al Pre-tratamiento de los efluentes, la segunda destinada al tratamiento físico-químico y la última para el acondicionamiento de lodos.

Palabras claves: Diseño de planta; Efluentes; Tratamiento; Reutilización

\begin{abstract}
In this article, the design and implementation of an industrial plant for the treatment of effluents generated in the production process of a tannery was developed in order to achieve its treatment and reuse in the activities of the company. Currently there are no major advances regarding the management of their effluents, being these directly disposed to the sewage system which has caused the breach of the environmental regulations in force in the country, causing a negative impact on the environment, in addition, it has been wasted the potential of reusing them within their processes. Due to this, it was decided to develop a technical study that allows to implement the proposed treatment plant according to the needs of the company. The treatment system is divided into three units, the first one for Pre-treatment of the effluents, the second one for the physical-chemical treatment and the last one for the conditioning of sludge.
\end{abstract}

Keywords: Plant design; Effluents; Treatment; Reuse 


\section{INTRODUCCIÓN}

La investigación realizada abarca el diseño e implementación de una Planta de Tratamiento de efluentes en una curtiembre. Actualmente el sector curtiembre juega un papel importante en la economía del país, siendo la producción de cuero según el INEI (2012) de 9,109 959.55 pies $^{2}$, con una proyección de crecimiento de $0.86 \%$ anual, sin embargo, existe una tendencia creciente al informalismo por lo que se estima que solo el $50 \%$ del cuero producido proviene de empresas formales (Rey, 2013). Cabe mencionar que las actividades de este sector durante años han venido generando un grave impacto ambiental negativo debido a la emisión de desechos sólidos (materia orgánica putrescible, residuos de descarne, recortes), gases tóxicos (VOCs del acabado, $\mathrm{H}_{2} \mathrm{~S}$ del pelambre y encalado; y $\mathrm{NH}_{3}$ del desencalado y purga) además de aguas residuales (con un alto contenido de materia oxidable y tóxica) (Martínez \& Romero, 2016) dentro de estos, causa especial interés la generación constante y en grandes volúmenes de efluentes debido que, presentan en su composición sustancias altamente contaminantes como DBO5, Cromo VI, N-NH4, DQO, sulfuros, sólidos suspendidos, grasas y aceites, coliformes fecales y elevados niveles de $\mathrm{pH}$. Además, estos efluentes tienen una disposición directa al alcantarillado sin contar con un tratamiento previo que los acondicione para tal fin, lo que ocasiona graves efectos sobre la vida acuática, las funciones naturales de los ríos, daños en la estructura de los suelos, la vegetación y la salud de las personas (Greenpeace, 2012).

Con el fin de mitigar los efectos perjudiciales provocados por las actividades de las empresas curtiembres, se promulgo el Decreto Supremo N $^{\circ} 003-2002-P R O D U C E$, en el cual se establecen los Límites máximos permisibles (LMP) para la emisión de efluentes en este sector industrial a partir de esto, se delimitó la concentración máxima que debe tener cada parámetro existente en su composición. Además, dentro del Decreto Supremo N¹7-2015- PRODUCE, se estableció la obligación de los titulares de cada empresa, a presentar un Plan de Manejo Ambiental el cual busca mediante acciones o inversiones lograr la reducción o eliminación de las sustancias contaminantes que se emiten al ambiente, dicha información, así como otros instrumentos de gestión ambiental deben ser remitidos al Organismo de Evaluación y Fiscalización Ambiental (OEFA). Sin embargo, a pesar de la legislación existente, la gran mayoría de las curtiembres no tienen adecuados sus procesos a las exigencias de estas normas además la informalidad existente en el sector contribuye a evadir el control ambiental establecido por el organismo pertinente.

Por lo tanto, a partir de la información recopilada acerca del tema; el cual viene siendo desarrollado desde hace algunos años en diferentes países; se ha observado distintos casos de éxito que demuestran la viabilidad y factibilidad de la implementación de una planta industrial que albergue un sistema de tratamiento de efluentes, es así que en el presente trabajo se decidió desarrollar un estudio técnico que permita el diseño de una planta industrial para el tratamiento de los efluentes generados en una curtiembre y su posterior recirculación en las actividades de la misma para esto, se tomó en cuenta la distribución actual del proceso productivo, el espacio disponible, los recursos existentes, entre otros aspectos. Además, se han utilizado técnicas de ingeniería, análisis de factores, diagramas, planos y cálculos que permitieron obtener como resultado final el dimensionamiento de las instalaciones de la planta.

\section{METODOLOGÍA}

La metodología desarrollada en el presente trabajo se basó en el estudio técnico realizado para el diseño de una planta industrial de tratamiento de efluentes.

\subsection{Localización de planta}

Se inició con el análisis de localización, la cual ya se encuentra definida debido que la planta de tratamiento será implementada en las instalaciones de la empresa por lo tanto, se procedió a determinar el área dentro de la misma que cuente con las condiciones adecuadas para su ubicación, se consideró que esta se ve comprometida a cumplir con algunos criterios establecidos en el Reglamento Nacional de Edificaciones

Nexo Revista Científica / Vol. 32, No. 02, pp. 166-178 / Diciembre 2019 
(norma OS.090) siendo principalmente, el distanciamiento respecto a las áreas administrativas (se debe evitar la interacción del personal con el área de tratamiento de efluentes, debido a la emisión de vapores y olores penetrantes) y a las de producción (debido a la generación de residuos o la posible ocurrencia de derrames que provocaría la contaminación del producto). Además se verificó que existe una escasa disponibilidad de áreas libres de trabajo dentro de la curtiembre. Por consiguiente, se concluyó que la única alternativa de ubicación dentro de la empresa que cumple con los parámetros descritos se encuentra en el primer nivel, junto a la zona de acabados, y a la zona de ingreso y descarga de materiales. Además, se justificó esta decisión a partir del análisis de factores realizado, en este se incluyó aquellos que tienen una relación directa con las características de la planta (Disponibilidad de espacio, costos administrativos y operativos; emisión y generación de residuos, y disposición de servicios básicos) como resultado se obtuvo que la ubicación escogida es la única área que tiene el potencial para albergar la planta de tratamiento al no entorpecer el normal desarrollo del proceso productivo así mismo, tiene habilitados los servicios básicos lo que facilita la conexión de equipos, por otro lado no genera mayores gastos adicionales para la instalación de equipos y la adecuación de ambientes y por último esta alejada de la zona administrativa de acuerdo a lo establecido en la norma mencionada anteriormente, con el fin de evitar cualquier efecto nocivo que puedan ocasionar los residuos u emisiones generadas por el funcionamiento de la planta de tratamiento.

\subsection{Tamaño de Planta}

Como segundo paso se desarrolló el análisis para el tamaño de planta, en este se consideró las relaciones existentes con cuatro factores considerados como influyentes en el tema: Recursos productivos, mercado, tecnología y financiamiento. Se concluyó que la principal consideración para determinar el tamaño de planta es el volumen de efluentes generados correspondiente a una producción de 300 a 450 cueros semanales $\left(74.18 \mathrm{~m}^{3}\right.$ a $\left.110.85 \mathrm{~m}^{3}\right)$, sin embargo se espera un crecimiento de la producción de acuerdo a su capacidad máxima instalada de 600 pieles semanales $\left(148.43 \mathrm{~m}^{3}\right)$ es así que la planta ha sido diseñada para albergar este volumen, por otro lado, no se ha considerado los otros factores como limitantes debido a que se dispone de los recursos productivos necesarios para el proceso así mismo se tiene identificado los recursos tecnológicos necesarios para la puesta en marcha de la planta de acuerdo a la capacidad total de tratamiento y por último, se dispone de los fondos económicos necesarios debido que el financiamiento proviene casi en su totalidad de fondos no reembolsables entregados por una entidad del estado.

\subsection{Definición del proceso}

El proceso fue dividido en tres unidades de tratamiento, la primera correspondiente al pre-tratamiento de efluentes que comprende desde el acopiamiento de efluentes tanto de pelambre, curtido como generales hasta la obtención de un efluente pre-tratado. En esta etapa, las aguas residuales provenientes de los botales de pelambre pasan por procesos secuenciales de filtración de residuos sólidos (pelos) y separación de grasas, el efluente filtrado es almacenado en un tanque para después tomar dos direcciones: pasar al proceso de oxidación; que tiene como objetivo cambiar el estado de oxidación de los sulfuros presentes para su posterior separación; o ser recirculado hacia los botales de pelambre, esto depende de las necesidades de producción. Finalmente, el efluente filtrado no recirculado (pre-tratado) es transferido a la segunda unidad de tratamiento. Para el pre-tratamiento de los efluentes generales, primero estos son recepcionados en una cámara de bombeo, luego son sometidos a procesos continuos de filtración de sólidos y de separación de grasas, por último, el efluente resultante (pre-tratado) es transferido a la siguiente unidad de tratamiento. Para el pre-tratamiento de los efluentes provenientes de los botales de curtido una vez que estos son recepcionados, pasan por un proceso de filtración de residuos sólidos, posteriormente son sometidos a un proceso de precipitación mediante la adición de Oxido de Magnesio, luego se dosifica manualmente Polielectrolita para acelerar el proceso de sedimentación, finalmente, el efluente sedimentado atraviesa un segundo proceso de filtración con el fin de lograr la separación del agua clarificada (efluente pre-tratado) de los lodos, el efluente resultante es transferido a la siguiente unidad de tratamiento, y los lodos son acumulados junto con otros residuos sólidos. 
La segunda unidad correspondiente al tratamiento físico - químico, abarca desde la recepción de efluentes pre-tratados hasta la decantación de los lodos existentes. Este proceso comienza con la recepción de los efluentes provenientes de la unidad anterior en un tanque de homogenización con el fin de igualar sus concentraciones luego, el efluente resultante, pasa por un proceso de coagulación mediante la adición de un reactivo coagulante (sulfato de amonio y agua) con el fin de desestabilizar el material contaminante para facilitar su posterior remoción y seguidamente pasa por un proceso de floculación mediante la adición de un reactivo floculante (Polielectrolita y agua) con el fin de aglomerar el material contaminante. A continuación, el efluente es sometido a un proceso de decantación en donde se separa la materia contaminante del efluente y así lograr su clarificación. Finalmente, el efluente tratado es recirculado hacia los procesos productivos iniciales.

La última unidad de tratamiento correspondiente al acondicionamiento de lodos, abarca desde la recepción de los lodos decantados hasta la acumulación de los lodos deshidratados es así que primero, los lodos provenientes de la etapa anterior son recepcionados en un tanque para luego pasar por un proceso de floculación en donde, mediante la adición de un reactivo floculante, estos son aglomerados para facilitar su posterior deshidratación, la cual tiene como objetivo disminuir los residuos de agua presentes en el lodo mediante un filtro de voluta, estos residuos son recirculados hacia el proceso de homogenización. El lodo deshidratado resultante es acumulado para su posterior tratamiento y disposición mediante una EPS (Empresa prestadora de servicios) al igual que los otros residuos sólidos generados durante el proceso.

\subsection{Distribución de planta}

Para este análisis se utilizó las herramientas de Tabla y Diagrama Relacional de Actividades, con el objetivo de determinar la disposición óptima y el ordenamiento de las diferentes áreas concernientes a la planta de tratamiento. La Tabla Relacional de Actividades permite visualizar las relaciones de cercanía entre cada actividad y evaluar la importancia de su proximidad. Para su desarrollo, se comenzó por evaluar las relaciones de acuerdo a los siguientes motivos: (1) Secuencia de flujo de trabajo, (2) Por abastecimiento de insumos, (3) No se desea la contaminación del lugar, (4) Por necesidad de tomar pruebas y monitoreo, (5) Seguridad y (6) No es necesario/ No guarda relación de actividades. De acuerdo a estos motivos se asignó la siguiente escala de valores para determinar el grado de cercanía que debe existir entre las actividades:

(A) "Absolutamente necesario", para aquellas actividades que utilizan los mismos equipos y que debido a la secuencia de proceso necesitan estar ubicadas juntas. (I) "Importante" para aquellas actividades no consecutivas pero que pertenecen a la misma unidad de tratamiento y pueden estar separadas por alguna actividad, (U) "Sin Importancia" para las actividades que no guardan relación con la secuencia del proceso y no es necesario que estén cerca y $(\mathrm{X})$ "No recomendable" para aquellas actividades que se considera inconveniente su cercanía, debido a que pueden representar un peligro para el personal o la producción habitual.

El análisis de cercanía de actividades desarrollado anteriormente, fue tomado como base para elaborar el Diagrama Relacional de Actividades, el cual permite observar gráficamente todas las actividades pertenecientes a la planta de tratamiento de acuerdo al grado de proximidad entre ellas, para esto se asignó a cada valor de proximidad un tipo de línea distintivo de acuerdo a los mostrado en la Tabla 2. Luego, se diagramó la distribución tentativa de la planta de tratamiento cumpliendo los criterios establecidos anteriormente (Figura 1). 


\section{G. Espinoza Gutiérrez et al.}

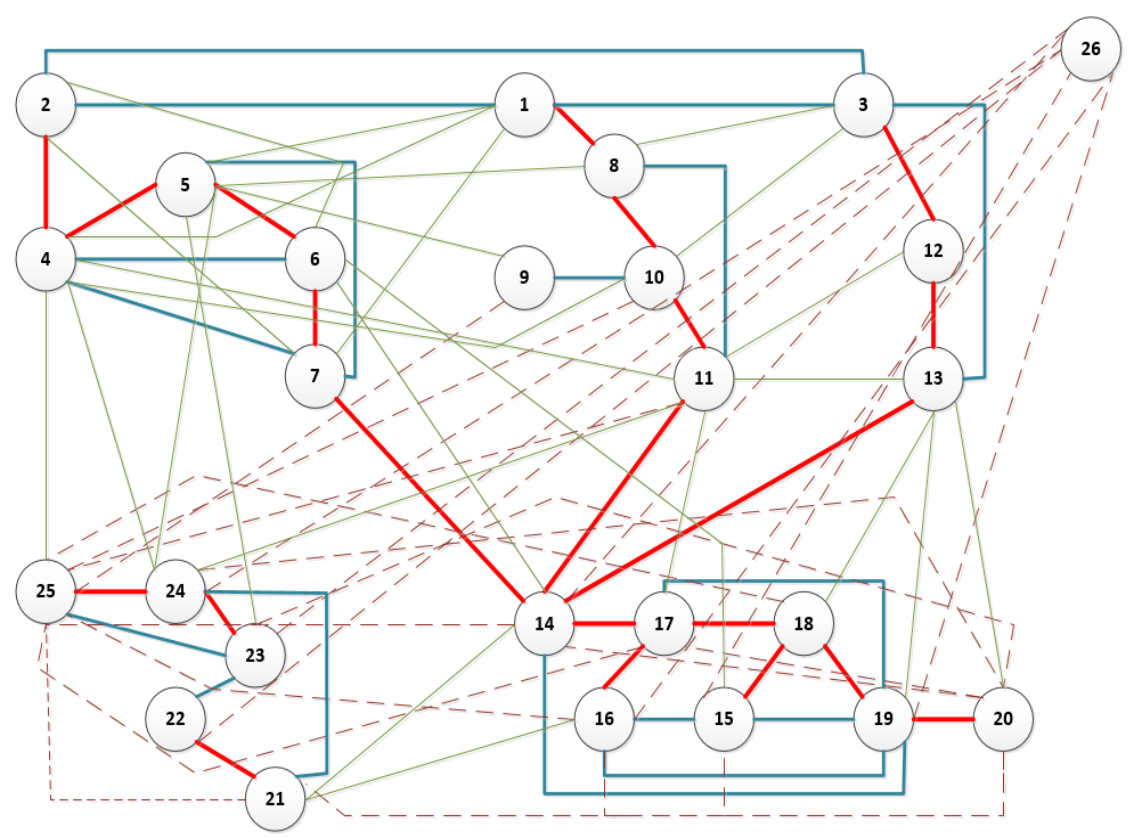

Figura 1. Diagrama Relacional de Actividades

Tabla 1. Leyenda de actividades - Diagrama Relacional de Actividades

\begin{tabular}{cccc}
\hline $\boldsymbol{N}^{\circ}$ & Actividad & $\boldsymbol{N}^{\circ}$ & Actividad \\
\hline 1 & Recepción de efluentes de curtido & 14 & Homogenización de efluentes pre-tratados \\
2 & Recepción de efluentes de Pelambre & 15 & Preparación de reactivo floculante \\
3 & Recepción de efluentes generales & 16 & Preparación de reactivo coagulante \\
4 & Filtración de residuos sólidos - Pelambre & 17 & Coagulación de efluentes pre-tratados \\
5 & Filtración de grasas - Pelambre & 18 & Floculación de efluentes pre-tratados \\
6 & Almacenamiento de efluente filtrado - Pelambre & 19 & Decantación de efluentes pre-tratados \\
7 & Oxidación de efluente filtrado - Pelambre & 20 & Disposición de efluentes tratados \\
8 & Filtración de residuos sólidos - Curtido & 21 & Almacenamiento de lodos \\
9 & Preparación manual de reactivos & 22 & Preparación de reactivo floculante de lodos \\
10 & Precipitación y sedimentación de efluente - Curtido & 23 & Floculación de lodos \\
11 & Filtración de efluente sedimentado - Curtido & 24 & Deshidratación de lodos \\
12 & Filtración de solidos finos de efluentes generales & 25 & Acumulación de lodos deshidratados \\
13 & Filtración de grasas - efluentes generales & 26 & Acumulación de residuos solidos \\
\hline
\end{tabular}

Tabla 2. Leyenda de líneas - Diagrama Relacional de Actividades

\begin{tabular}{ccc}
\hline Código & Valor de Proximidad & Tipo \\
\hline A & Absolutamente necesario & \\
I & Importante & \\
U & Sin importación & \\
X & No recomendable & ------ \\
\hline
\end{tabular}

Además se desarrolló un análisis para determinar la mano de obra necesaria en el funcionamiento de la planta, en este caso no se consideró conveniente aplicar la técnica de balance de línea debido que el funcionamiento de la planta es automatizado y por lo tanto, no requiere la presencia permanente de operarios que trabajen con los equipos sin embargo, si es necesario la supervisión periódica para el desarrollo de algunas actividades, es por ello que en la Tabla 3 se decidió identificarlas y señalar la labor de verificación

Nexo Revista Científica / Vol. 32, No. 02, pp. 166-178 / Diciembre 2019 
que se realizan en cada una de ellas. De acuerdo a lo mostrado, se determinó que solo es necesaria la presencia de un operario por los lapsos de tiempo consignados para las actividades de supervisión y eventualmente un operario adicional para la preparación de reactivos. Además de acuerdo a la experiencia de la empresa consultora lo recomendable es solo mantener la cantidad señalada de personal.

Tabla 3. Supervisión de Actividades

\begin{tabular}{|c|c|c|c|c|}
\hline \multirow{2}{*}{ Descripción } & \multicolumn{2}{|c|}{ Supervisión } & \multirow{2}{*}{$\begin{array}{l}\text { T. de Supervisión } \\
\text { (min) }\end{array}$} & \multirow{2}{*}{ Actividad } \\
\hline & SI & NO & & \\
\hline $\begin{array}{l}\text { Filtración de residuos sólidos del efluente } \\
\text { (Pelambre) }\end{array}$ & & $\mathrm{X}$ & & \\
\hline $\begin{array}{l}\text { Filtración de grasas del efluente } \\
\text { (Pelambre) }\end{array}$ & & $\mathrm{X}$ & & \\
\hline $\begin{array}{l}\text { Almacenamiento de efluente filtrado } \\
\text { (Pelambre) }\end{array}$ & $\mathrm{X}$ & & 10 & $\begin{array}{l}\text { Verificar valores de transmisor e } \\
\text { flujo y de } \mathrm{pH}\end{array}$ \\
\hline Oxidación del efluente filtrado (Pelambre) & & $\mathrm{X}$ & & \\
\hline $\begin{array}{l}\text { Filtración de residuos sólidos del efluente } \\
\text { (Curtido) }\end{array}$ & & $\mathrm{X}$ & & \\
\hline $\begin{array}{l}\text { Preparación manual de reactivo (Oxido de } \\
\text { Magnesio) }\end{array}$ & $\mathrm{X}$ & & 10 & $\begin{array}{l}\text { Preparación de Oxido de } \\
\text { Magnesio para precipitación }\end{array}$ \\
\hline Precipitación del efluente (Curtido) & $\mathrm{X}$ & & 5 & $\begin{array}{c}\text { Dosificación manual de reactivo } \\
\text { a tanque }\end{array}$ \\
\hline $\begin{array}{l}\text { Preparación manual del reactivo } \\
\text { (Polielectrolita) }\end{array}$ & $\mathrm{X}$ & & 10 & $\begin{array}{c}\text { Preparación de Polielectrolita } \\
\text { para sedimentación }\end{array}$ \\
\hline Sedimentación del efluente (Curtido) & $\mathrm{X}$ & & 5 & $\begin{array}{l}\text { Dosificación manual de reactivo } \\
\text { a tanque }\end{array}$ \\
\hline $\begin{array}{l}\text { Filtración del efluente sedimentado } \\
\text { (Curtido) }\end{array}$ & & $\mathrm{X}$ & & \\
\hline $\begin{array}{l}\text { Filtración de solidos finos del efluente } \\
\text { (Efluentes generales) }\end{array}$ & & $\mathrm{X}$ & & \\
\hline $\begin{array}{c}\text { Filtración de grasas del efluente (Efluentes } \\
\text { generales) }\end{array}$ & & $\mathrm{X}$ & & \\
\hline Homogenización de efluentes pre-tratados & $\mathrm{X}$ & & 10 & $\begin{array}{l}\text { Verificar valores de transmisor } \\
\text { de flujo y de } \mathrm{pH}\end{array}$ \\
\hline Preparación de reactivo coagulante & $\mathrm{X}$ & & 10 & $\begin{array}{l}\text { Adición de reactivo líquido a } \\
\text { tanque de coagulante }\end{array}$ \\
\hline Coagulación de efluentes pre-tratados & & $\mathrm{X}$ & & \\
\hline Preparación de reactivo floculante & $\mathrm{X}$ & & 20 & $\begin{array}{l}\text { Adición de insumo químico y } \\
\text { agua fresca /Control de reacción }\end{array}$ \\
\hline Floculación de efluentes pre-tratados & & $\mathrm{X}$ & & \\
\hline Decantación de efluentes pre-tratados & $\mathrm{X}$ & & 10 & $\begin{array}{l}\text { Verificar valores de transmisor } \\
\text { de flujo y } \mathrm{pH}\end{array}$ \\
\hline Almacenamiento de lodos & & $\mathrm{X}$ & & \\
\hline $\begin{array}{l}\text { Preparación de reactivo floculante de } \\
\text { lodos }\end{array}$ & $\mathrm{X}$ & & 20 & $\begin{array}{l}\text { Adición de insumo químico y } \\
\text { agua fresca, control de reacción }\end{array}$ \\
\hline Floculación de lodos & & $\mathrm{X}$ & & \\
\hline Deshidratación de lodos & & $\mathrm{X}$ & & \\
\hline
\end{tabular}

Posteriormente, para determinar el área requerida por los espacios físicos de la planta se decidió utilizar el método de Guerchet. Según este método para el cálculo de la superficie total de planta, se necesita la suma de los siguientes elementos:

- Superficie estática (Ss): Es la superficie que ocupa físicamente el equipo 
- Superficie gravitacional (Sg): Es la superficie utilizada por los operarios que están trabajando con el equipo.

- Superficie de evolución (Se): Es la superficie necesaria para el movimiento, material y medios de transporte.

Para desarrollar esta herramienta primero se identificó el número total de equipos (elementos estáticos) así como sus dimensiones y se consideró el número de operarios determinados anteriormente para identificar los elementos móviles. Luego, para realizar el cálculo de cada tipo de superficie es necesario considerar un coeficiente de evolución (K), el cual fue obtenido de la siguiente manera:

$$
\begin{gathered}
K=\frac{\text { Altura media de elementos moviles }}{2 * \text { Altura media de elementos estáticos }} \\
\qquad \begin{array}{c}
K=\frac{1.65}{2 * 1.3} \\
K=0.63
\end{array}
\end{gathered}
$$

Una vez obtenido el valor de K, se calculó la Superficie total (St), para esto fue necesario sumar los valores obtenidos de la Superficie estática, gravitacional y de evolución, los resultados obtenidos se muestran en la Tabla 4.

Tabla 4. Calculo de Superficie Total

\begin{tabular}{lcccc}
\hline \multicolumn{1}{c}{ Elementos Estáticos } & $\begin{array}{c}\boldsymbol{S} \boldsymbol{s}\left(\boldsymbol{m}^{2}\right) \\
\boldsymbol{L x} \boldsymbol{A}\end{array}$ & $\begin{array}{c}\boldsymbol{S g}\left(\boldsymbol{m}^{2}\right) \\
\boldsymbol{S} \boldsymbol{x} \boldsymbol{N}\end{array}$ & $\begin{array}{c}\boldsymbol{S e}\left(\boldsymbol{m}^{2}\right) \\
(\boldsymbol{S s}+\boldsymbol{S g}) \boldsymbol{K}\end{array}$ & $\begin{array}{c}\boldsymbol{S t}\left(\boldsymbol{m}^{2}\right) \\
(\boldsymbol{S s}+\boldsymbol{S g}+\boldsymbol{S e}) \boldsymbol{Q}\end{array}$ \\
\hline Filtro de discos & 0.97 & 0.97 & 1.22 & 3.15 \\
Cámara de bombeo & 8.85 & 8.85 & 11.21 & 28.90 \\
Filtro de pelos & 1.15 & 1.15 & 1.46 & 3.76 \\
Tanque de tratamiento de curtido & 2.91 & 2.91 & 3.69 & 9.52 \\
Trampa de grasas (Pelambre) & 2.50 & 2.50 & 3.17 & 8.17 \\
Trampa de grasa (Efluentes generales) & 4.5 & 4.50 & 5.70 & 14.70 \\
Tanque de efluentes de Pelambre & 6.63 & 6.63 & 8.39 & 21.64 \\
Soplador para oxidación & 0.02 & 0.02 & 0.03 & 0.07 \\
Tanque de oxidación de sulfuros & 7.82 & 7.82 & 9.91 & 25.55 \\
Mezclador estático & 0.02 & 0.00 & 0.01 & 0.04 \\
Tanque de coagulante & 3.30 & 3.30 & 4.18 & 10.78 \\
Tanque de floculante & 3.30 & 3.30 & 4.18 & 10.78 \\
Bomba dosificadora electrónica & 0.02 & 0.02 & 0.03 & 0.06 \\
Tanque homogenizador & 26.22 & 26.22 & 33.22 & 85.66 \\
Soplador para homogenizador & 0.02 & 0.02 & 0.02 & 0.06 \\
Tanque sedimentador & 58.39 & 58.39 & 73.89 & 190.75 \\
Bomba de lodos & 0.05 & 0.00 & 0.03 & 0.07 \\
Bomba dosificadora & 0.02 & 0.02 & 0.03 & 0.06 \\
Tanque de almacenamiento de lodos & 1.69 & 1.69 & 2.14 & 5.53 \\
Tanque de floculante (lodos) & 1.26 & 1.26 & 1.59 & 4.11 \\
Tanque floculador de lodos & 1.69 & 3.38 & 3.22 & 8.29 \\
Filtro de voluta & 0.42 & 0.42 & 0.53 & 1.38 \\
$\quad$ Elementos Móviles & & & & \\
Operarios & & & & 4.9 \\
TOTAL & 0.5 & 1 & 0.95 & $\mathbf{4 3 7 . 9 3}$ \\
\hline
\end{tabular}


De acuerdo a los cálculos desarrollados para determinar la superficie total (Tabla 4), se concluye que el área aproximada que requiere la planta de tratamiento es de $497.93 \mathrm{~m}^{2}$.

Por ultimo para el diseño de instalaciones eléctricas se determinó que es necesario implementar un nuevo tablero de distribución cercano a la planta de tratamiento de efluentes, esto con el fin de reducir la caída de tensión, perdidas de energía y costos de implementación que provocaría la instalación de varios alimentadores. El cálculo de la máxima demanda proyectada (Tabla 5) se realizó en base a lo indicado en el Código Nacional de Electricidad - Utilización y además se consideró el número de equipos requeridos y la potencia de cada uno consignada por el fabricante.

Tabla 5. Calculo de la máxima demanda proyectada

\begin{tabular}{ccccc}
\hline Descripción & Cant. & $\begin{array}{c}\text { Carga/Uni. } \\
(\boldsymbol{k W})\end{array}$ & $\boldsymbol{F . S .}$ & $\boldsymbol{M D}(\boldsymbol{k W})$ \\
\hline Soplador & 2 & 14.914 & 0.6 & 17.90 \\
Bomba Sumergible & 4 & 1.119 & 0.6 & 2.68 \\
Bomba de lodos & 1 & 1.119 & 0.6 & 0.67 \\
Agitador Floculante & 2 & 0.186 & 0.6 & 0.22 \\
Agitador Coagulante & 1 & 0.186 & 0.6 & 0.11 \\
Agitador Floculante de lodos & 1 & 0.186 & 0.6 & 0.11 \\
Dosificador coagulante & 1 & 0.373 & 0.6 & 0.22 \\
Dosificador floculante & 1 & 1.119 & 0.6 & 0.67 \\
Dosificador floculante para lodos & 1 & 0.373 & 0.6 & 0.22 \\
Válvula de Lodos & 1 & 0.075 & 0.6 & 0.04 \\
Agitador Cromo & 1 & 0.373 & 0.6 & 0.22 \\
Máxima demanda total (kW) & & & & $\mathbf{2 3 . 0 9}$ \\
\hline
\end{tabular}

Por otro lado, para el desarrollo de este análisis se determinó un factor de simultaneidad de encendido de las máquinas de $60 \%$. De acuerdo a lo obtenido en la tabla anterior se concluye que la máxima demanda total proyectada para la planta de tratamiento es de $23.09 \mathrm{~kW} / \mathrm{h}$.

\section{RESULTADOS Y DISCUSIÓN}

De acuerdo al análisis desarrollado para el diseño de una planta de tratamiento de efluentes se obtuvieron los siguientes resultados:

La ubicación seleccionada es el único espacio disponible dentro de la curtiembre que tiene el potencial para albergar la planta de tratamiento, no representa altos costos administrativos y operativos para su instalación, se encuentra alejada de la zona administrativa con el fin de evitar cualquier riesgo al personal, además se dispone de los servicios básicos de agua y electricidad por lo que solo serán necesarios trabajos para la conexión de equipos.

Se ha definido el proceso preliminar de tratamiento, este ha sido dividido en tres unidades: Pre-tratamiento, Tratamiento físico-químico y Acondicionamiento de lodos.

La capacidad óptima para la planta de tratamiento (tamaño de planta), se ha determinado en base a las consideraciones analizadas anteriormente, por lo tanto, el volumen de efluentes a tratar será de $10 \mathrm{~m}^{3} / \mathrm{h}$ correspondiente al volumen de descarga debido a la capacidad máxima instalada de producción.

Nexo Revista Científica / Vol. 32, No. 02, pp. 166-178 / Diciembre 2019 
Para el desarrollo de la distribución tentativa de la planta de tratamiento de efluentes se ha utilizado las herramientas de Tabla Relacional, Diagrama Relacional de Hilos y el método Guerchet, en base a consideraciones iniciales, como la secuencia de trabajo, la interacción de actividades, dimensiones de equipos, la normativa correspondiente y los ambientes de trabajo.

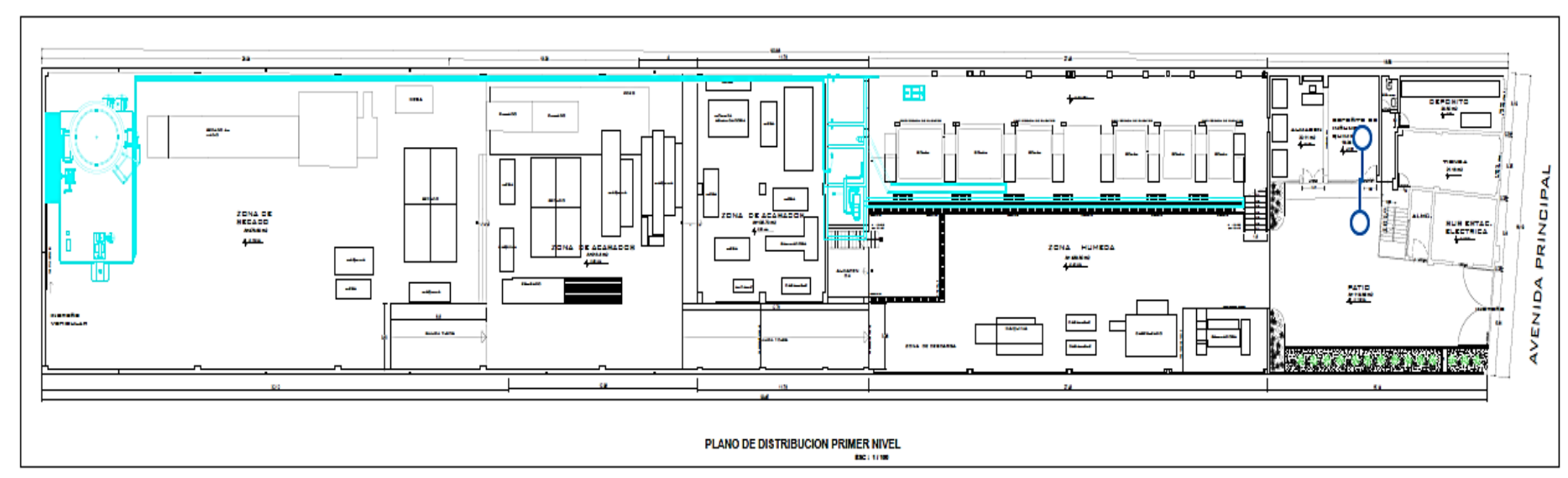

Figura 2. Layout preliminar de la planta de tratamiento de efluentes

Para la elaboración de los planos de las instalaciones eléctricas se tomó como base lo establecido en el Código Nacional de Electricidad - Utilización, además, se consideró el cálculo realizado anteriormente para determinar la máxima demanda total proyectada correspondiente a $29.03 \mathrm{~kW} / \mathrm{h}$. Por lo tanto, se ha requerido la instalación de un nuevo tablero de distribución cercano a la ubicación escogida, para evitar posibles caídas de tensión y así mismo la instalación de un tablero de control para el funcionamiento automatizado de la planta de tratamiento, en las Figuras 4 y 5 se observan las conexiones eléctricas para los equipos, tomacorrientes interruptores y luminarias.

\begin{tabular}{|l|c|l|}
\hline \multicolumn{1}{|c|}{ ESPECIFICACIÓN } & SÍMBOLO & $\begin{array}{l}\text { ALTURA } \\
\text { SNPT }(\mathbf{m})\end{array}$ \\
\hline TABLERO DE DISTRIBUCION & & \\
\hline SALIDA PARA CENTRO DE LUZ & & 1.80 SNPT. \\
\hline TOMACORRIENTE TRIFASICO CON TOMA A TIERRA & & TECHO \\
\hline INTERRUPTOR DOBLE & - & 0.40 SNPT. \\
\hline PUESTA A TIERRA & & 1.20 SNPT. \\
\hline RED POR TECHO-CIRCUITO ALUMBRADO & & PISO (PATIO) \\
\hline RED POR PISO-CIRCUITO TOMACORRIENTES & & PISO O TECHO \\
\hline CONDUCTOR PARA PUESTA A TIERRA & & PISO \\
\hline RED POR PISO-CIRCUITO TOMACORRIENTES & & PISO \\
\hline
\end{tabular}

Figura 3. Leyenda de símbolos para planos eléctricos 


\section{G. Espinoza Gutiérrez et al.}

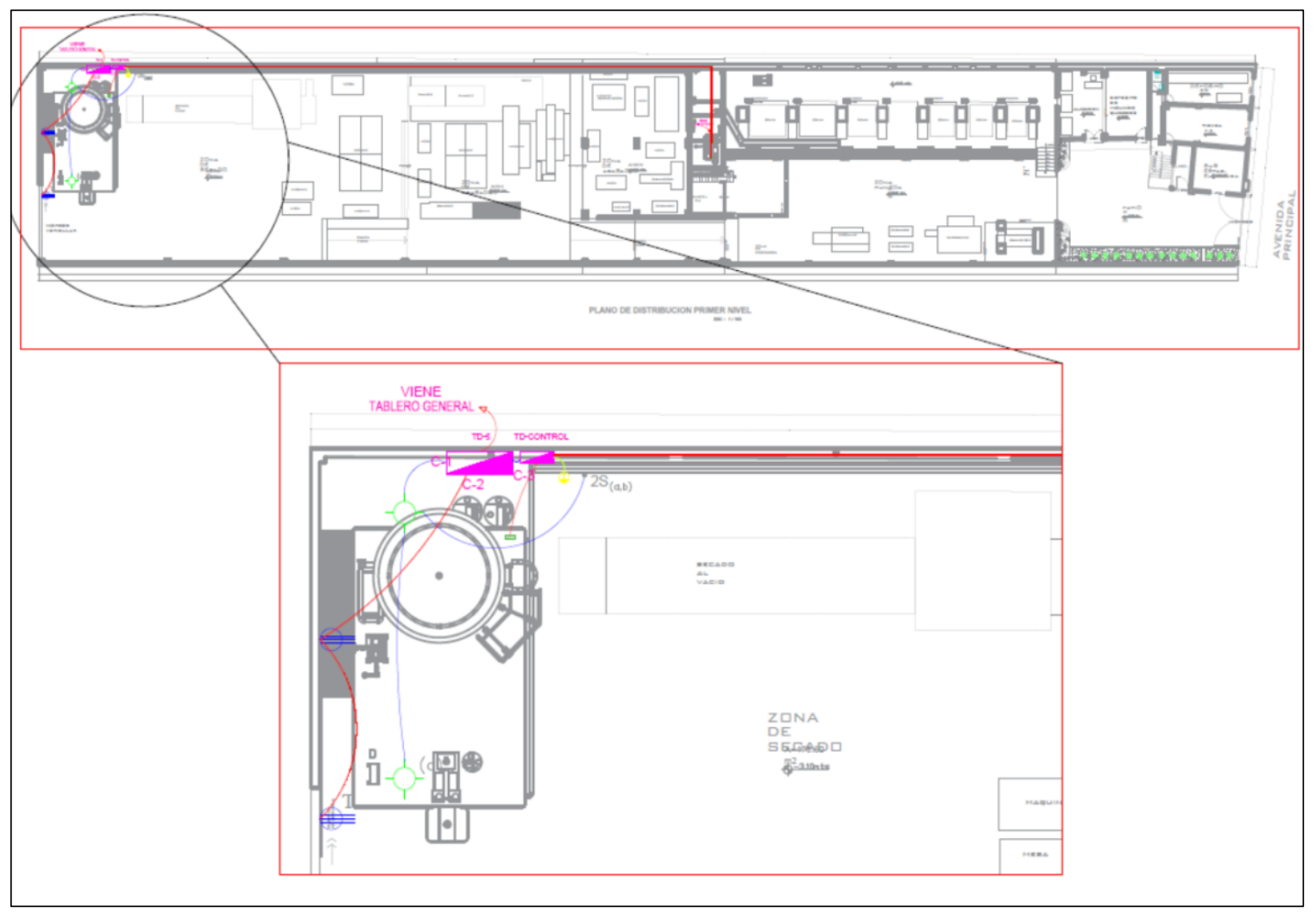

Figura 4. Plano de instalaciones eléctricas - A

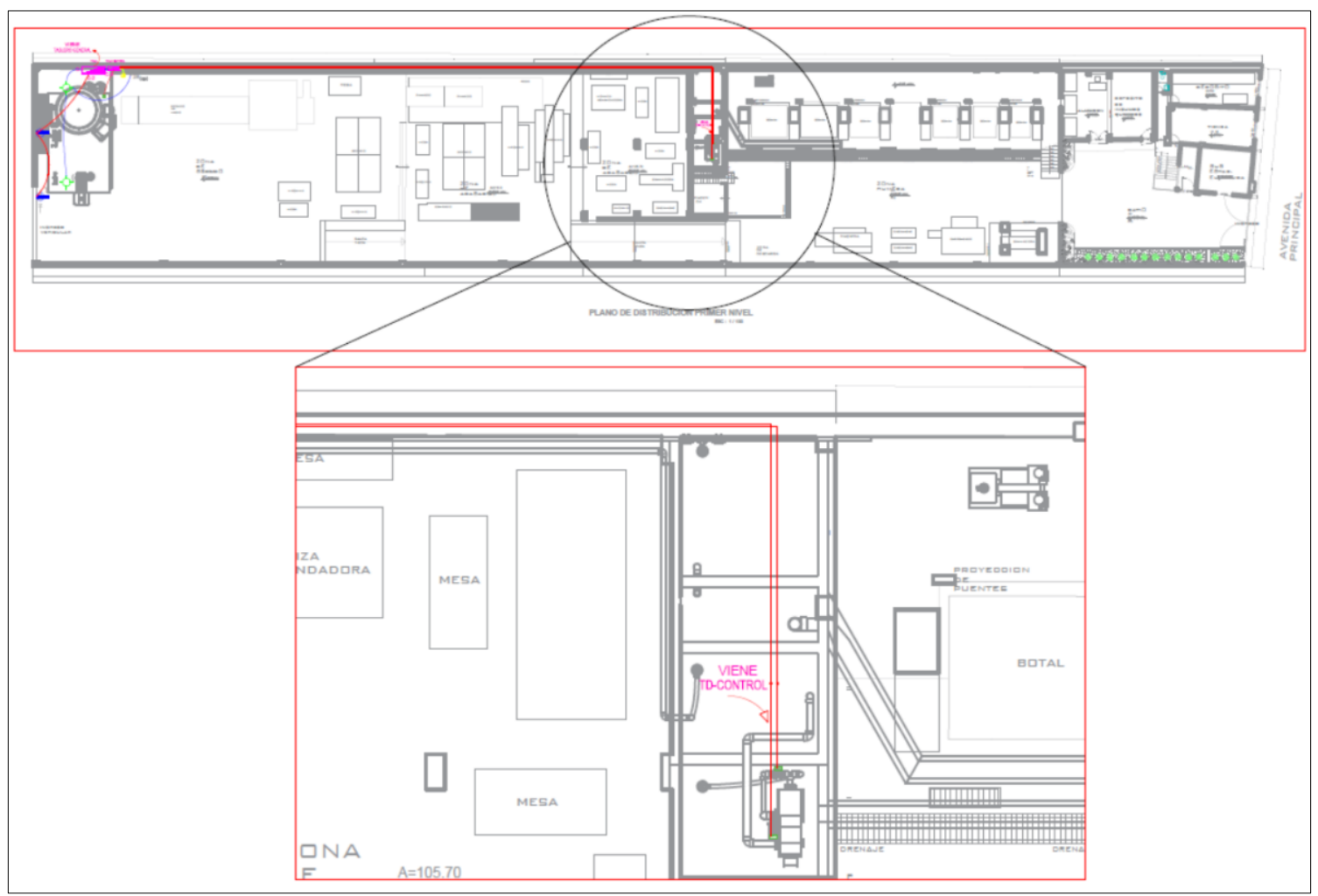

Figura 5. Plano de instalaciones eléctricas - B 


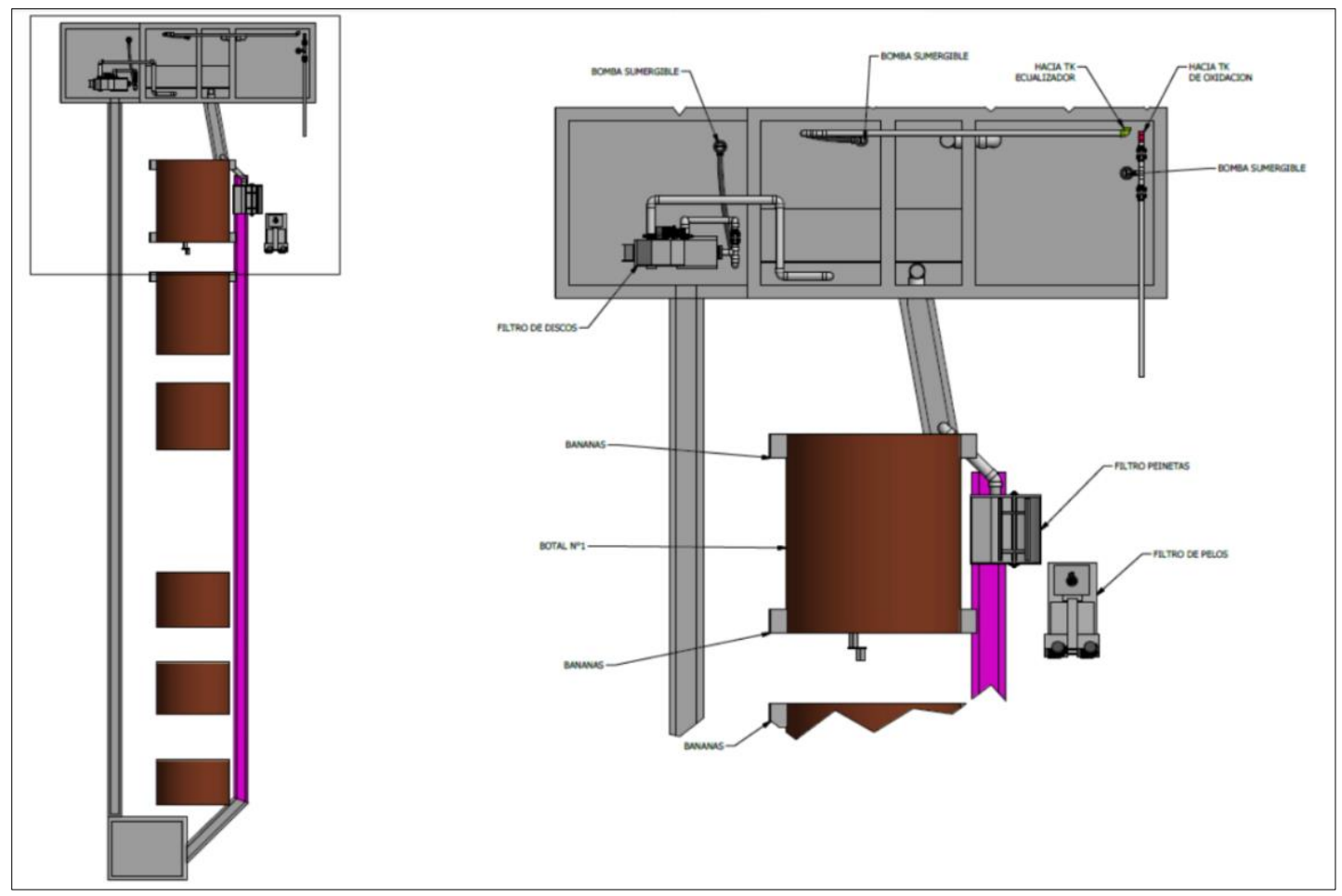

Figura 6. Modelamiento planta de tratamiento - Pretratamiento de efluentes

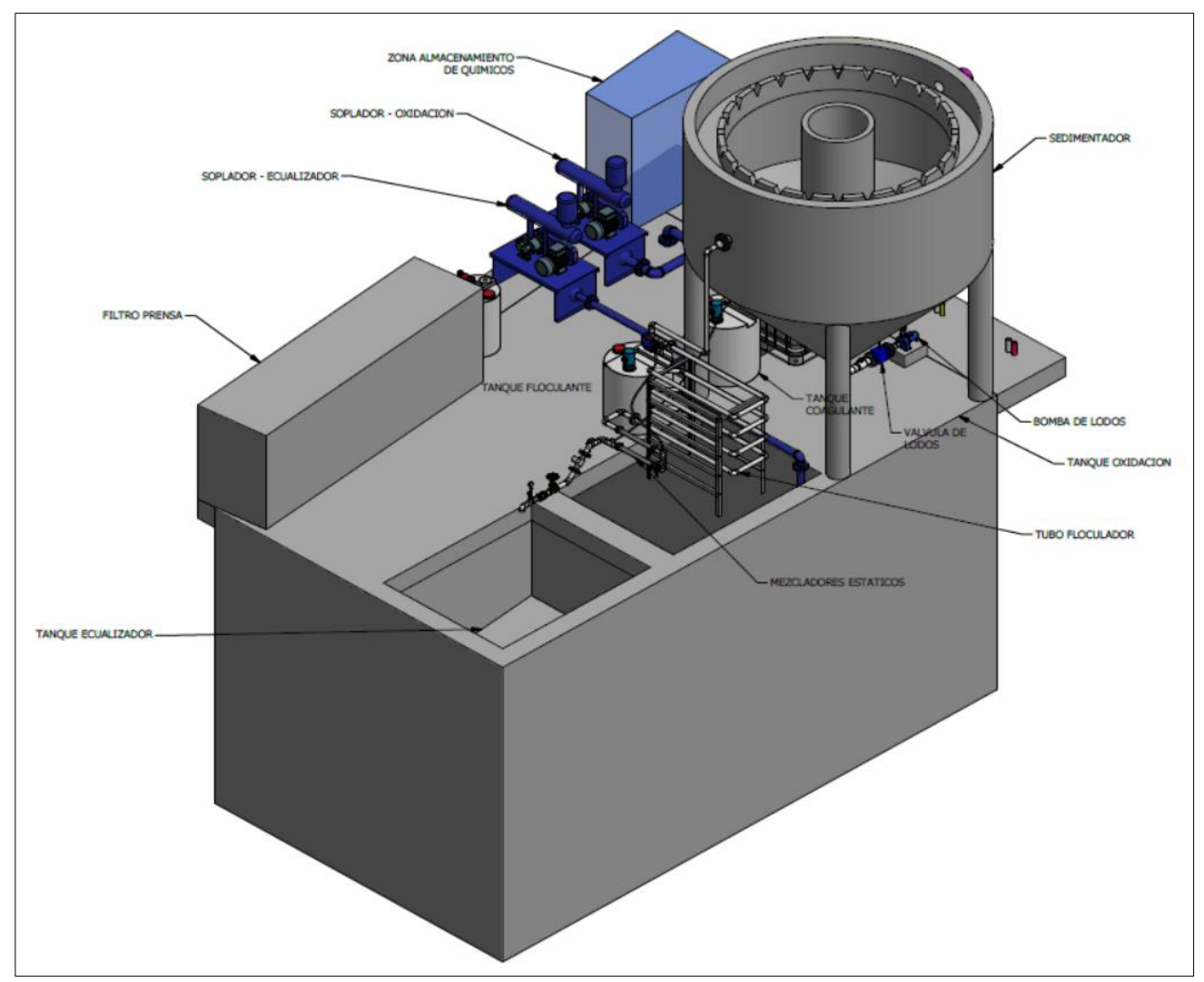

Figura 7. Modelamiento planta de tratamiento - Tratamiento fisicoquímico y acondicionamiento de lodos 
G. Espinoza Gutiérrez et al.

Mediante el software de diseño Autodesk Inventor se ha realizado el modelamiento 3D de la planta de tratamiento de efluentes, considerando las dimensiones reales del proyecto tanto en la unidad de pretratamiento de efluentes, cercana a los botales, como en las demás unidades de tratamiento. Las figuras 6 y 7 muestran el resultado de este modelamiento.

\section{CONCLUSIONES}

A partir del estudio realizado se ha comprobado que para la elaboración correcta de un diseño de planta es indispensable la utilización de herramientas de ingeniería como el análisis de factores para determinar la localización y el tamaño de planta; método de Guerchet para determinar el área de trabajo requerida y el método de Muther para desarrollar la distribución de planta.

Para el dimensionamiento de la planta fue importante tener como base un proceso definido para el tratamiento de los efluentes generados en la curtiembre, así como las dimensiones de los equipos necesarios de acuerdo a la capacidad de tratamiento esperada.

Se determinó que el área necesaria para la implementación de la planta de tratamiento de efluentes es de $497.93 \mathrm{~m}^{2}$ además para realizar las conexiones eléctricas de los equipos es necesario habilitar un nuevo tablero de distribución trifásico cercano a la ubicación de la planta con el fin de evitar caídas de tensión o costos adicionales de instalación.

La planta de tratamiento de efluentes ha sido diseñada para albergar la descarga total de los mismos generados por la producción 600 cueros semanales correspondiente a capacidad máxima instalada en la empresa.

\section{REFERENCIAS}

Aprueban Límites Máximos Permisibles y Valores Referenciales para las actividades industriales de cemento, cerveza, curtiembre y papel - Decreto Supremo N ${ }^{\circ} 003$ - 2002- PRODUCE. (19 de agosto de 2002). Diario Oficial El Peruano, pp. 230921- 230925.

Aprueban el Reglamento de Gestión Ambiental para la Industria Manufacturera y Comercio InternoDecreto Supremo No17-2015- PRODUCE. (6 de junio de 2015). Diario Oficial El Peruano, pp.554437554449

Autoridad Nacional del Agua (2009). Evaluación de la eficiencia del uso del agua en la cuenca del Rio Chili. Recuperado de http://www.ana.gob.pe/normatividad/evaluacion-de-la-eficiencia-del-uso-del-aguaen-la-cuenca-del-rio-chili-0

Fernández, J. (2015). Diseño de un sistema de tratamiento de aguas residuales para optimizar el proceso de pelambre en la empresa "Curtipiel Martinez", Parroquia Izamba, cantón Ambato, Provincia de Tungurahua, Periodo 2013-2014 (Tesis de pregrado). Universidad Técnica de Cotopaxi, Latacunga, Ecuador. Recuperado de http://repositorio.utc.edu.ec/handle/27000/2746

Garay, B., (2007). Disposición de Planta. Lima, Perú: Fondo Editorial Universidad de Lima. 
G. Espinoza Gutiérrez et al.

Greenpeace (2013) La contaminación de las curtiembres. Cueros Tóxicos II. Recuperado de https://www.greenpeace.org/argentina/Global/argentina/report/2013/contaminacion/Cueros-toxicos-IIGreenpeace.pdf

Martínez, S., \& Romero J. (2016). Revisión del estado actual de la industria de las curtiembres en sus procesos y productos: Un análisis de su competitividad. Scientific Electronic Library Online, 26(1), 113124. doi: $10.18359 /$ rfce. $2357 \mathrm{gr}$

Organismo de Evaluación y Fiscalización Ambiental (2014). Supervisión a empresas. Recuperado de https://www.oefa.gob.pe/actividades-principales/supervision-a-empresas-2

Salas, C. (2005). Reúso de cromo en el tratamiento de efluentes de una curtiembre. Revista Peruana de Química e Ingeniería Química. 8(2), pp. 61-64.

Valdés, D. (2012). Diseño preliminar de un sistema de tratamiento de aguas residuales a escala industrial para los efluentes del procesamiento de pieles (Tesis de pregrado). Universidad San Francisco de Quito, Quito, Ecuador. Recuperado de http://repositorio.usfq.edu.ec/handle/23000/1881

Zeballos, J. (2014). Evaluación de la contaminación ambiental generada por efluentes industriales en el proceso productivo de una curtiembre de mediana capacidad del parque industrial de Rio seco, Arequipa (Tesis de pregrado). Universidad Nacional de San Agustín, Arequipa, Perú.

\section{SEMBLANZA DE LOS AUTORES}

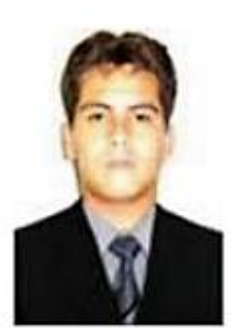

José A. Aguilar Franco: Obtuvo el grado de Ingeniero Industrial en la Universidad Católica San Pablo, Perú, donde actualmente es profesor titular, además de Ingeniero de Investigación del Programa de Iniciación Científica, formulador de proyectos y Coordinador General del Instituto de Energía y Medio Ambiente de la Universidad Católica San Pablo. Desarrolló sus estudios de maestría en la Pontificia Universidad Católica de Río de Janeiro (PUC - Río) y Universidad Nacional de San Agustín, Perú (UNSA). Ha desarrollado una estancia de investigación en Italia para Capacitación en Uso de Maquinarias Ecológicas. Cuenta con experiencia como asesor de Tesis, además trabaja como Formulador de Proyectos bajo la modalidad de concurso a nivel Nacional en proyectos de Investigación e Innovación convocadas por el Estado Peruano.

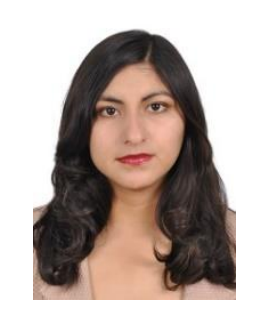

Gisel Espinoza Gutiérrez: Bachiller de la carrera profesional de Ingeniería Industrial en la Universidad Católica San Pablo, Perú. Actualmente viene desarrollando su tesis en el Instituto de Energía y Medio Ambiente (IEM) de la Universidad Católica San Pablo para obtener el título profesional de Ingeniero Industrial.

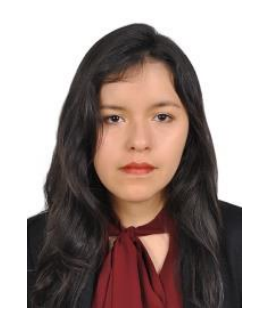

Paola F. Barreda Cuellar: Bachiller de la carrera profesional de Ingeniería Industrial en la Universidad Católica San Pablo, Perú. Actualmente viene desarrollando su tesis en el Instituto de Energía y Medio Ambiente (IEM) de la Universidad Católica San Pablo para obtener el título profesional de Ingeniero Industrial. 